\title{
Prevalencia y manejo de condilomas en población consultante en Chile: estudio "DIACON"
}

\author{
Andrea Schilling R. ${ }^{1}$, Andrea Huneeus V. ${ }^{1}$, Alejandra Massoc P. ${ }^{2}$, \\ Francisca Rivera M. ${ }^{2}$ y Gabriel Cavada Ch. ${ }^{3}$
}

\section{Prevalence and management of condylomas in consulting population in Chile: "DIACON study"}

Introduction: Condylomas or genital warts (GW) are the most frequently diagnosed sexually transmitted infection (STI) in STI centers in Chile, but there are no population statistics available. Objectives: To describe the prevalence of GW in patients from 18-60 years of age who attend outpatient dermatology, gynecology and urology practice; the demographic characteristics of the patients and the diagnostic and treatment tools. Methods: A sample of Chilean specialists stratified by region, population and gender of patients was provided with a logbook and answered a questionnaire. Results: The GW prevalence was $2.44 \%$ for the whole group; $3.76 \%$ for the $18-34$ age group and $1.29 \%$ for the $35-60$ years group $(\mathrm{p}=0.0000)$. The average age of patients with GW was 29.4 years in women and 32.7 years in men $(\mathrm{p}=0.019)$. The distribution by age was different according to gender and health system. Visual inspection was the most frequent diagnostic method used and imiquimod cream the most common treatment, however, there were differences in the use of diagnostic and therapeutic tools according to the patient's gender, specialty of the doctor and health system. Conclusions: The high prevalence of GW confirmed the need and importance of public health interventions to address this problem.

Keywords: Condylomas; genital warts; prevalence; distribution.

Palabras clave: Condilomas; verrugas genitales; prevalencia; distribución.

\section{Introducción}

L os condilomas o verrugas genitales (VG) son tumoraciones benignas que se observan como pequeños bultos de color carne con apariencia similar a la coliflor, en la piel y las membranas mucosas de los genitales en hombres y mujeres, causados por el virus del papiloma humano (VPH). Alrededor de 30 millones de casos nuevos se diagnostican cada año en todo el mundo ${ }^{1}$. Sin embargo, los datos de incidencia por país y las estimaciones de prevalencia varían ampliamente según la población y el diseño del estudio, y van desde 1,4\% en España a 25,6\% en Nigeria ${ }^{2,3}$. En América Latina, los estudios de prevalencia han arrojado resultados que van desde 0,4\% en mujeres en Buenos Aires, Argentina, hasta $3,9 \%$ en mujeres en Porto Alegre, Brasil; y de 3,6\% entre los hombres sin infección por VIH que asisten a un centro de infecciones de transmisión sexual (ITS) en la Ciudad de México, a 7,8\% entre los hombres co-infectados por VIH en el mismo centro ${ }^{2,4-5}$.

Las únicas estadísticas sobre VG en Chile provienen del Programa de Infecciones de Transmisión Sexual (ITS) en el Sistema Público de Salud. En el año 2008, las VG representaron $41,3 \%$ de los diagnósticos de ITS, siendo la ITS más diagnosticada en dichos centros? Desde entonces, se ha observado un aumento sostenido en los ingresos mensuales al programa de ITS del sistema público de salud por dicha patología: 4.521 pacientes el año 2012, 7.497 pacientes el año 2014 y 10.349 pacientes el año 2016, constituyendo actualmente $51,8 \%$ de las ITS notificadas en dichos centros ${ }^{8}$.

Giuliano y cols. diseñaron un estudio observacional para estimar la carga de enfermedad para VG que se aplicaría a dermatólogos, ginecólogos y urólogos en forma de encuesta telefónica o en línea y una bitácora diaria de 10 días para el registro de pacientes ambulatorios de 18 a 60 años utilizando la misma metodología en diferentes países ${ }^{9}$. Los primeros resultados de este protocolo ya se han informado para Polonia, con una prevalencia de consulta para VG de $2,9 \%{ }^{10}$, Taiwán, con una prevalencia de $1,1 \%{ }^{11}$, y para los Emiratos Árabes Unidos y el Reino de Arabia Saudita con una prevalencia de $0,01 \%{ }^{12}$. Con el acceso a la traducción al español hecha por Salmeron ${ }^{13}$, adaptamos esta encuesta para ser utilizada con médicos chilenos. Nuestro estudio tuvo como objetivo describir la incidencia y la prevalencia de VG en pacientes de 18 a 60 años que consultaran en forma ambulatoria a dermatología, ginecología y urología, y describir los
${ }^{1}$ Facultad de Medicina Clínica Alemana-Universidad del Desarrollo.

${ }^{2}$ MSD, Santiago, Chile. ${ }^{3}$ Unidad de Investigación Clínica Alemana y Facultad de Medicina Universidad Finis Terrae.

Conflictos de Interés. Andrea Schilling recibió financiamiento para este estudio de MSD-Chile $y$ ha recibido honorarios por estudios clínicos relacionados con vacunas contra el VPH de Merck \& Co. Andrea Huneeus y Bioestadístico Gabriel Cavada no presentan conflicto de intereses que declarar. Alejandra Massoc es empleada de MSD Chile y es posible que posea acciones $\mathrm{y} / \mathrm{u}$ opciones sobre acciones en la compañía. Francisca Rivera fue el Punto de Contacto Designado de MSD y podría tener acciones propias y/u opciones sobre acciones en la compañía. El financiamiento para este estudio fue proporcionado por MSD Chile, Santiago Chile, a través de Merck \& Co.

Recibido: 29 de enero de 2018 ( $1^{\circ}$ versión), 18 de enero de 2019 (versión definitiva) Aceptado: 27 de enero de 2019

Correspondencia a: Andrea Schilling Redlich dra.andrea.schilling@gmail.com 
datos demográficos por región geográfica y por sistema de salud público o privado. Además, quisimos describir las prácticas actuales en el diagnóstico y tratamiento de VG y establecer si existían diferencias según sexo del paciente, según especialidad del médico y entre el sistema de salud público y privado, con respecto a las herramientas de diagnóstico y tratamiento. Los resultados de este estudio aportarían información actualizada sobre VG y establecerían una base sobre la cual comparar los datos epidemiológicos en el futuro, midiendo el impacto de la vacuna contra el VPH sobre dicha patología.

\section{Materiales y Métodos}

Estudio observacional, en forma de encuesta telefónica o en línea, y un registro diario de pacientes por 10 días, aplicado a una muestra aleatoria de médicos. Se utilizó el acrónimo DIACON (DIAgnóstico CONdilomas) para todos los documentos y la correspondencia con los participantes por la facilidad de ser recordado, y es el nombre bajo el cual se han divulgado resultados parciales en congresos desde entonces.

\section{Muestra}

Estuvo conformada por dermatólogos, ginecólogos y urólogos pertenecientes a la lista de acceso público de la Superintendencia de Salud de Chile (SS) y de la Corporación Nacional Autónoma de Certificación de Especialidades Médicas (CONACEM) al 30 de junio de 2013, quienes fueron invitados a participar por medio de un correo electrónico. Con base en los datos descritos para

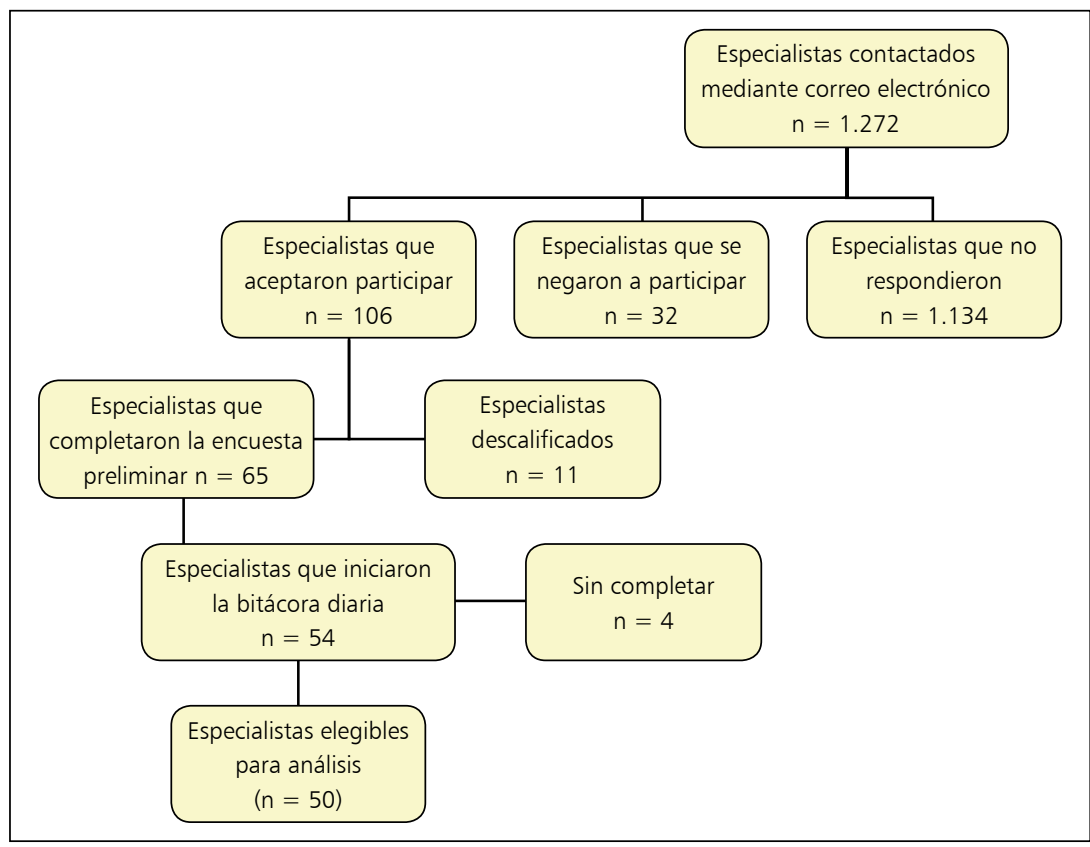

Figura 1.
América Latina ${ }^{5,6}$ asumimos una prevalencia de VG de $1 \%$. Para nuestro análisis, se requería un mínimo de 1.522 pacientes ambulatorios atendidos durante el período de 10 días (IC del 95\%, margen de error de $0,5 \%$ ), lo que equivalía encuestar a 26 médicos con un flujo promedio de 30 pacientes atendidos por semana. Sin embargo, decidimos incluir 50 médicos para la encuesta para dar cuenta de los que no respondieran. Considerando que el Departamento del Programa Nacional para la Prevención y el Control del VIH/SIDA y las ITS en Chile estimaba que $65 \%$ de los diagnósticos de VG se realizaría en mujeres y $35 \%$ en hombres ${ }^{14}$, la muestra se construyó con $55 \%$ de médicos especialistas que atienden a mujeres (ginecólogos), 25\% de especialistas que atienden preferentemente a hombres (urólogos) y $20 \%$ de especialistas que atienden pacientes de ambos sexos (dermatólogos). Teniendo en cuenta las diferencias geográficas del país y la proporción de la población nacional en ellas, la muestra también se estratificó por área geográfica. Se invitó a participar a 1.272 especialistas cuyas direcciones de correo electrónico fue posible rastrear entre todos los dermatólogos, ginecólogos y urólogos mostrados en la lista de acceso público de la SS y CONACEM al 30 de junio de 2013 ( $n=252,1.131$ y 247, respectivamente, total 1.630). Entre los que respondieron a la invitación, se seleccionó una muestra aleatoria, estratificada por área geográfica y por especialidad. Se realizó una encuesta preliminar de los criterios de inclusión y exclusión. La muestra final incluyó a 10 dermatólogos, 27 ginecólogos y 13 urólogos, quienes participaron en el estudio entre el 3 de diciembre de 2013 y el 2 de abril de 2014 (Figura 1).

\section{Criterios de inclusión}

Para participar, los médicos debían estar certificados en dermatología, obstetricia-ginecología o urología; tener entre 2 y 30 años de práctica desde que completaron la especialización; dedicar al menos $50 \%$ de su tiempo a atender pacientes ambulatorios; tener un mínimo de 30 consultas ambulatorias en una semana típica, con al menos $50 \%$ de los pacientes ambulatorios entre las edades de 18 y 60 años; y haber dado su consentimiento informado para la participación.

\section{Medidas}

Los médicos participantes recibieron una bitácora diaria para registrar las características demográficas de los pacientes que consultaran durante un período de trabajo de 10 días. El último día se administró una encuesta para investigar las herramientas de diagnóstico y tratamiento para VG en sus últimos 10 pacientes con dicha patología, tanto en práctica pública como privada. Estos 10 últimos pacientes no necesariamente tenían que haber sido atendidos durante el período de tiempo registrado en la bitácora diaria, también podían haber sido atendidos previamente. 


\section{Definiciones}

Para el registro de los pacientes que consultaran por condilomas, se definió como casos nuevos aquellos en que el paciente nunca había sido diagnosticado de condilomas previamente y casos previos si habían sido diagnosticados por el médico del estudio u otro médico en forma previa. Además, estos últimos se clasificaron como casos recurrentes si se trataba de un segundo o más episodio diagnosticado por el médico del estudio u otro médico, y como caso resistente si no habían respondido a tratamiento.

Dos comités de ética locales (Comité de Ética Científica Facultad de Medicina Clínica Alemana-Universidad del Desarrollo y Comité de Ética Científica Servicio de Salud Metropolitano Oriente) dieron su aprobación tanto al protocolo como a la encuesta. Un equipo de organización de investigación ( $\mathrm{CRO}$, por sus siglas en inglés) con sede en Santiago fue responsable de ponerse en contacto con los médicos, controlar la recopilación de datos y guiar el proceso de encuesta auto administrada por teléfono.

\section{Análisis estadístico}

Las variables cuantitativas se describieron mediante promedio y desviación estándar, mientras que las variables categóricas mediante frecuencias y porcentajes. La comparación de prevalencias e incidencias por grupo etario se realizó mediante un análisis de regresión de Poisson. La comparación de las proporciones de uso de las herramientas diagnósticas y terapéuticas por sexo, especialidad y por sistema de atención se realizó mediante el test de diferencia de proporciones basado en la distribución normal y la diferencia distribucional de variables se docimó con la prueba de KolmogorovSmirnov. La prevalencia se calculó como el número de pacientes con VG dividido por el número total de pacientes vistos durante los 10 días de registro por los médicos de la muestra, expresados en términos de porcentaje y su respectivo intervalo de confianza. La incidencia se calculó como el número de casos nuevos dividido por el número total de pacientes atendidos durante los 10 días de registro. Se utilizó un nivel de significación de 5\% y los intervalos de confianza son de nivel $95 \%$. Se consideró como significativo un $p<0,05$. Los datos se analizaron utilizando el software STATA versión 14.0

\section{Resultados}

\section{Tasa de respuesta}

Ciento seis médicos fueron elegibles para participar, y se construyó una muestra aleatoria estratificada de 65 . De éstos, 11 no cumplieron con los criterios de inclusión y cuatro no completaron la encuesta; por lo tanto, la muestra estudiada consistió en 50 especialistas ( 27 ginecólogos, 13 urólogos y 10 dermatólogos) (Figura 1).
Los datos demográficos de los médicos participantes se muestran en la Tabla 1.

\section{Registro diario de los pacientes}

El número total de pacientes atendidos durante el período de 10 días fue de 8.780: 4.120 en el rango de 18-34 años y 4.660 en el rango de 35-60 años. Fueron atendidos por dermatólogos 1.639 pacientes $(18,7 \%)$, 5.019 pacientes por ginecólogos $(57,2 \%)$ y 2.122 pacientes por urólogos $(24,2 \%)$.

\section{Prevalencia e incidencia de las verrugas genitales}

Durante el período de 10 días, se observaron 215 pacientes con VG. La prevalencia de VG fue de 2,4\%; (IC $95 \% 2-2,8 \%$ ) (215/8.780 pacientes). En el grupo de 18-34 años se observaron $155 \mathrm{VG}$, lo que dio una prevalencia de 3,7\% (155/4.120), y en el grupo de 35 a 60 años se observaron $60 \mathrm{VG}$, lo que dio una prevalencia de 1,2\% (60/4.660). La razón de prevalencia de tener VG es 2,94 veces mayor en el grupo de edad de 18-34 años vs. el grupo de 35 a 60 años $(p=0,0000)$.

Los dermatólogos atendieron a $22,8 \%$ de los pacientes con VG (49/215), los ginecólogos atendieron a $58,6 \%$ de los pacientes con VG (126/215) y los urólogos a 18,6\%. (40/215). La prevalencia no difirió según la especialidad (dermatología: 3,0\%, ginecología: $2,5 \%$ y urología: 1,9\%) $(p=0,086)$. Tampoco hubo diferencias estadísticamente significativas por región geográfica.

En 45,6\% de los pacientes las VG eran casos nuevos y $54,4 \%$ fueron casos previos. Entre los casos previos,

Tabla 1. Características demográficas de los 50 médicos participantes en el estudio

Características demográficas

Edad promedio

$48,4 \pm 9,23$ años

Sexo

$68 \%$ masculino / $32 \%$ femenino

Tiempo de ejercicio promedio desde

titulado en la especialidad

$17,5 \pm 9,29$ años

Entorno laboral

$100 \%$ Urbano

Sistema de salud ${ }^{(1)}$

50\% Público y Privado / 50\% sólo Privado

Muestra estratificada por especialidad, distribución geográfica y distribución porcentual de la población del país por área

$\begin{array}{lccccc}\text { Región geográfica } & \text { \% Población del país } & \text { GINE }(n) & \text { URO (n) } & \text { DERM (n) } & \text { Total(2) }^{(2)} \\ \text { Norte } & 12,37 & 4 & 1 & 1 & 6 \\ \text { Centro } & 55,99 & 14 & 8 & 6 & 28 \\ \text { Sur } & 31,64 & 9 & 4 & 3 & 16 \\ \text { Total } & 100,00 & 27 & 13 & 10 & 50\end{array}$

Abreviaturas: GINE = ginecólogos; URO = urólogos; DERM = dermatólogos. ${ }^{(1)}$ Cada médico podía trabajar en más de un sistema. ${ }^{(2)}$ Total de médicos encuestados. 
en $17,7 \%$ se trató de condilomas recurrentes y $2,3 \%$ eran resistentes.

Hubo 98 nuevos casos de VG, lo que constituye una incidencia de 98 casos nuevos en 8.780 pacientes consultantes a dermatólogos, ginecólogos y urólogos en 10 días, es decir 11 casos nuevos de VG por cada 1.000 consultantes de 18 a 60 años atendidas por dermatólogos, ginecólogos y urólogos en 10 días. En el grupo de pacientes consultantes de 18-34 años se observaron 70 casos nuevos de VG, lo que dio una incidencia de 70 casos nuevos en 4.120 pacientes consultantes a dermatólogos, ginecólogos y urólogos en 10 días, es decir 17 casos nuevos de VG por cada 1.000 consultantes de 18 a 34 años atendidos por dermatólogos, ginecólogos y urólogos en 10 días. En el grupo de pacientes consultantes de 35 a 60 años se observaron 28 casos nuevos de VG, lo que dio una incidencia de 28 casos nuevos en 4.660 pacientes consultantes a dermatólogos, ginecólogos y urólogos en 10 días, es decir seis casos nuevos de VG por cada 1.000 consultantes de 34 a 60 años atendidos por dermatólogos, ginecólogos y urólogos en 10 días.
La incidencia es 2,86 veces mayor en el grupo de 18 a 34 años que en el de 35 a 60 años $(p=0,0000)$. Esta incidencia no difirió según la especialidad o región geográfica.

El 75,3\% de los pacientes con VG se atendió en el sistema privado de salud.

El $64,7 \%$ de los pacientes diagnosticados con $\mathrm{VG}$ fueron mujeres.

Los urólogos vieron más casos nuevos que los otros especialistas: E1 62,5\% de sus pacientes con VG fueron casos nuevos, $53,1 \%$ de los vistos por dermatólogos y $37,3 \%$ de los vistos por ginecólogos $(\mathrm{p}=0,010)$.

La edad media de los pacientes con VG fue de 30,6 años (rango: 18 a 58 años). Las mujeres con VG fueron más jóvenes (media = 29,4 años; rango de 18 a 57 años) que los hombres con VG (media = 32,7 años; rango de 18 a 58 años $)(p=0,019)$. La distribución por edad y sexo se muestra en la Figura 2a. La edad media de los pacientes con VG tratadas en un hospital público fue 27,4 años (rango: 18 a 55 años) y en la práctica privada 31,6 años (rango: 18 a 58 años) $(\mathrm{p}=0,003)$. La distribución según edad y sistema de salud se muestra en la Figura $2 b$.
Figura 2. (a) Distribución por edad y sexo, pacientes de 18 a 60 años con condilomas. Estudio DIACON Chile. (b) Distribución por edad y sistema de salud, pacientes de 18 a 60 años con condilomas. Estudio DIACON Chile.

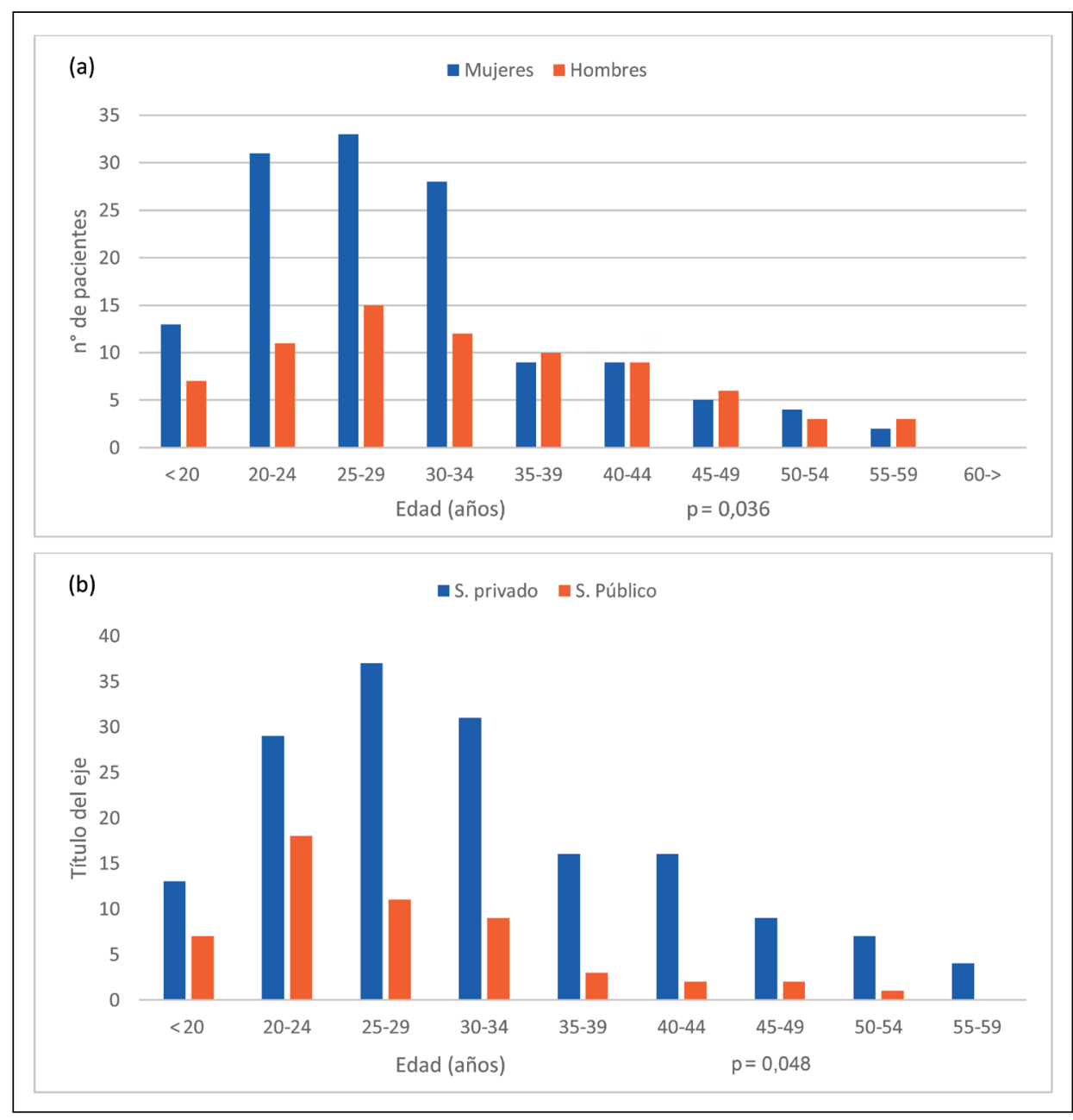




\section{Utilización de los recursos de atención médica}

Principales herramientas diagnósticas: La inspección visual se utilizó en $66,7 \%$ de las ocasiones, seguido de métodos de aumento como colposcopia/anoscopia/uretroscopia en $13,3 \%$, pruebas de ácido acético en $13,1 \%$, biopsia en $6,1 \%$, reacción de polimerasa en cadena (RPC) en $0,5 \%$. (Se podía usar más de un método diagnóstico). En las mujeres se usaron más las pruebas de ácido acético y en los hombres la biopsia y RPC (Tabla 2a). Aunque la inspección visual fue la herramienta de diagnóstico más utilizada por las tres especialidades, hubo diferencias importantes en la distribución del uso de las diferentes herramientas según especialidad. Los ginecólogos usan significativamente más métodos de magnificación que los dermatólogos (colposcopia) $(\mathrm{p}=0,003)$, los dermatólogos más inspección visual tanto en mujeres $(\mathrm{p}=0,0016)$ como en hombres $(\mathrm{p}=0,0000)$ y los urólogos realizan más biopsias que los dermatólogos (p 0,0000).
Respecto al uso de herramientas diagnósticas según tipo de servicio de salud, no hubo diferencias en su uso según fuera servicio público o privado.

Recursos terapéuticos. Para el tratamiento de las verrugas genitales, la crema de imiquimod auto-aplicada fue la indicación más común (33,0\%), seguida de la aplicación de podofilina al $10-30 \%$ por el médico $(25,3 \%)$, la crioterapia $(16,1 \%)$ y la aplicación de ácido tricloro-acético al $80-90 \%$ por parte del médico $(14,2 \%)$. Se prescribió podofilotoxina al $0,5 \%$ auto-aplicada al $6,9 \%$ de los pacientes y la escisión quirúrgica se realizó en 4,2\%.

En las mujeres se indicó más ácido tricloro-acético y en los hombres más podofilina y crioterapia (Tabla 2b). En el sistema privado se indicó más imiquimod y en el sistema público se indicó más podofilina, crioterapia y cirugía (Tabla 3 ).

Según especialidad médica, los ginecólogos usan

Tabla 2. Principales herramientas diagnósticas y terapéuticas utilizadas según sexo del paciente

\begin{tabular}{|c|c|c|c|c|c|}
\hline & \multicolumn{2}{|c|}{ Mujeres } & \multicolumn{2}{|c|}{ Hombres } & \multirow[t]{2}{*}{ Valor $p$} \\
\hline & $\mathbf{n}$ & $\%$ & $\mathbf{n}$ & $\%$ & \\
\hline \multicolumn{6}{|l|}{ (a) Principales herramientas diagnósticas $(n=1.203)$ * } \\
\hline Inspección visual & 463 & 66,5 & 340 & 67,1 & NS \\
\hline Métodos de magnificación** & 99 & 14,2 & 62 & 12,2 & NS \\
\hline Tests de ácido acético & 125 & 18,0 & 33 & 6,5 & 0,0000 \\
\hline Biopsia & 8 & 1,2 & 66 & 13,0 & 0,0000 \\
\hline RPC & 1 & 0,1 & 6 & 1,2 & 0,0192 \\
\hline \multicolumn{6}{|l|}{ (b) Principales herramientas terapéuticas $(n=887)$} \\
\hline Imiquimod crema auto-aplicada por el paciente & 179 & 34,7 & 114 & 30,7 & NS \\
\hline Podofilina $10-30 \%$ aplicada por el médico & 93 & 18,0 & 132 & 35,6 & 0,0000 \\
\hline Crioterapia & 82 & 15,9 & 61 & 16,4 & NS \\
\hline Ácido triciclo-acético 80-90\% aplicado por el médico & 116 & 22,5 & 10 & 2,7 & 0,0000 \\
\hline Podofilotoxina $0,5 \%$ auto-aplicada por el paciente & 33 & 6,4 & 29 & 7,8 & NS \\
\hline Cirugía & 13 & 2,5 & 25 & 6,7 & 0,0000 \\
\hline
\end{tabular}

*Podía utilizarse más de una herramienta diagnóstica. **Métodos de Magnificación en mujeres: colposcopía. En hombres: anoscopía, meatoscopía, uretroscopía. RPC: reacción de polimerasa en cadena.

Tabla 3. Herramientas terapéuticas utilizadas por sistema de salud y sexo del paciente

\begin{tabular}{|c|c|c|c|c|c|}
\hline & $\begin{array}{c}\text { Sistema } \\
\mathbf{n}\end{array}$ & $\begin{array}{l}\text { de Salud } \\
\%\end{array}$ & $\begin{array}{c}\text { Sistema } \\
\mathbf{n}\end{array}$ & $\begin{array}{l}\text { de Salud } \\
\%\end{array}$ & Valor $p$ \\
\hline (a) Mujeres $(n=516)$ & \multicolumn{2}{|c|}{$(n=150)$} & \multicolumn{2}{|c|}{$(n=366)$} & \\
\hline Imiquimod crema auto-aplicada por el paciente & 32 & 21,3 & 147 & 40,2 & 0,0000 \\
\hline Ácido triciclo-acético 80-90\% aplicado por el médico & 34 & 22,7 & 82 & 22,4 & NS \\
\hline Podofilina $10-30 \%$ aplicada por el médico & 31 & 20,7 & 62 & 16,9 & NS \\
\hline Crioterapia & 25 & 16,7 & 57 & 15,6 & NS \\
\hline Podofilotoxina $0,5 \%$ auto-aplicada por el paciente & 26 & 17,3 & 7 & 1,9 & 0,0000 \\
\hline Cirugía escisional & 2 & 1,3 & 11 & 3,0 & NS \\
\hline (b) Hombres ( $\mathrm{n}=371$ ) & \multicolumn{2}{|c|}{$(n=166)$} & \multicolumn{2}{|c|}{$(n=205)$} & Valor $\mathrm{p}$ \\
\hline Podofilina $10-30 \%$ aplicada por el médico & 65 & 39,157 & 67 & 32,682 & NS \\
\hline Imiquimod auto-aplicada por el paciente & 20 & 12,048 & 94 & 45,853 & 0,0000 \\
\hline Crioterapia & 42 & 25,301 & 19 & 9,269 & 0,0000 \\
\hline Podofilotoxina $0,5 \%$ auto-aplicada por el paciente & 10 & 6,024 & 19 & 9,269 & NS \\
\hline Cirugía & 21 & 12,651 & 4 & 1,952 & 0,0000 \\
\hline Ácido tricloro-acético 80-90\% aplicado por el médico & 8 & 4,819 & 2 & 0,975 & 0,0230 \\
\hline
\end{tabular}


signifcativamente más ácido tricloro-acético al 80-90\% $(\mathrm{p}=0,0000)$, los dermatólogos la crioterapia tanto en mujeres como hombres $(\mathrm{p}=0,0000)$ los urólogos la podofilina $10-30 \%$ aplicada por el médico $(\mathrm{p}=0,0002)$ y la cirugía $(\mathrm{p}=0,0000)$.

El número de visitas entre el diagnóstico y el fin del tratamiento durante un episodio de VG fue mayor para pacientes del sistema privado que para los del sistema público, tanto para hombres $(2,95$ vs $1,75, \mathrm{p}=0,0026)$ como para mujeres $(3,51$ vs $1,43 \mathrm{p}=0,001)$.

\section{Discusión}

La alta prevalencia de VG en pacientes ambulatorios de 18-60 que consultaron a dermatólogos, ginecólogos y urólogos en Chile es consistente con la prevalencia de VG publicada por estudios basados en exámenes genitales. Patel y cols., describen en su revisión sistemática (Estudios de Europa, Norte-América, Asia, Sud-América y Australia) un rango de prevalencia en este tipo de estudios entre 0,2 y $5,1 \%{ }^{2}$. Los estudios de prevalencia para América Latina en dicha revisión sistemática sólo incluían datos de Argentina y Brasil, y eran del tipo de auto reporte de condilomas en los últimos 12 meses o de por vida, con una prevalencia de $1,2 \%$, y se trataba de pacientes que participan en programas de detección precoz del cáncer cervico-uterino ${ }^{4,6}$. Sin embargo, al comparar con la publicación de Parkin y cols. ${ }^{5}$, que incluye en su publicación a México y más países de Centroamérica y Sudamérica, la prevalencia es similar a la descrita en nuestro estudio. Ambos autores explican que las estimaciones de prevalencia tienden a ser más altas entre los estudios basados en el examen genital que en los de revisión de fichas o auto reporte. En nuestro estudio, el diagnóstico también se basó en examen genital. Respecto a la diferencia de prevalencia por grupo etario se corresponde con lo descrito en la literatura médica y la revisión de Patel y cols., ya que en la mayoría de las series se muestra una máxima carga de enfermedad entre los 20 y 35 años ${ }^{2}$.

A diferencia de otras ITS como gonorrea, sífilis, hepatitis B y VIH/SIDA que son más frecuentes en la región norte de $\mathrm{Chile}^{14}$, no se encontró evidencia de asociación entre diagnóstico de VG y región geográfica en este estudio. Esto podría significar que la muestra no sea representativa de las regiones o que las VG tienen un comportamiento epidemiológico diferente a las demás ITS en nuestro país, lo que requiere de mayor estudio y análisis. En una tesis de grado recientemente presentada, respecto a pacientes mujeres que acudieron a un policlínico de condilomas en el Hospital Padre Hurtado en el área suroriente de Santiago, los factores de riesgo para su presencia y respuesta al tratamiento se relacionaron más con morbilidad general que con factores tradicionalmente relacionados a ITS. ${ }^{15}$
Las tasas de incidencia anual reportadas en la literatura médica internacional oscilan entre 118 y 205 casos nuevos por cada 100.000 personas en población general ${ }^{2}$. En nuestro estudio no fue posible calcular la incidencia poblacional, ya que se desconoce el porcentaje de la población que consulta a dermatólogos, ginecólogos y urólogos en nuestro país. Se pudo realizar una estimación más gruesa mediante el cálculo de incidencia de casos nuevos que lograron ser atendidos por especialistas en el año de estudio. Para esto se usó la cifra de la población total susceptible como denominador de la tasa poblacional. Según las proyecciones censales ${ }^{16}$, el año 2014 la población de Chile de 18-60 años estaba constituida por 10.815.915 habitantes. Considerando que los 50 médicos participantes constituían 3\% de los especialistas (50/1.630), la proporción de la población que atenderían equivaldría a $3 \%$ de la demanda poblacional $=324.477$ habitantes. La incidencia de casos nuevos que lograron ser atendidos por especialistas en un año, equivaldría a $98 \mathrm{VG} / 324.477$ habitantes $=30$ cada 100.000 habitantes año. Esta incidencia probablemente sea sólo una fracción pequeña de la incidencia real poblacional, porque existen importantes barreras de acceso a la consulta por ITS. Se han descrito barreras personales, como la baja auto-conciencia de examen genital, la falta de percepción o conocimiento de las personas sobre las VG y la vergüenza; culturales, como la existencia de remedios caseros o naturales y el miedo a la estigmatización; socioeconómicas como la baja disponibilidad de especialistas o la mala distribución poblacional de éstos y el costo de las atenciones ${ }^{17,18}$. Una de las barreras e inequidades de salud descritas en Chile es el acceso a las especialidades médicas, con una importante gradiente a favor de los quintiles de mayor ingreso CASEN para la consulta por especialista. En el año 2013 sólo 9,3\% de los usuarios de FONASA vs $18,3 \%$ de los usuarios de ISAPRE utilizaron el recurso en un tiempo dado ${ }^{19,20}$.

Además de la barrera económica previamente descrita, que $75,3 \%$ de los pacientes con VG haya sido atendido en la práctica privada también puede deberse a que la muestra no fue estratificada por tipo de sistema de salud, resultando en una mayor proporción de médicos atendiendo en el sistema privado.

En nuestro estudio, la edad promedio de los pacientes con condilomas (30,4 años) fue mayor que la edad media reportada a nivel mundial, pero esto puede explicarse porque en la bitácora diaria se incluían sólo pacientes que tenían entre 18 y 60 años, mientras que otras publicaciones incluyen pacientes más jóvenes ${ }^{2}$. Sin embargo, tal como se describe en la literatura científica, la edad media de las mujeres con VG fue menor que la de los hombres, y la distribución por edad de los pacientes también fue diferente entre los sexos ${ }^{2}$. La menor edad media de los pacientes atendidos en el sistema de salud pública frente 
a el sistema privado y la diferencia en la distribución de edad por sistema de salud podría explicarse por diferentes motivos. Al igual que en la literatura médica mundial, se ha reportado para Chile que los jóvenes que buscan atención en los establecimientos de salud pública tienen un mayor riesgo de contraer una ITS debido, en parte, a un menor uso de condones ${ }^{21,22}$. Alternativamente, podría ser que los jóvenes de más recursos económicos de nuestro país, al tener un seguro de salud administrado por sus padres, consulten menos, ya que significaría que tendrían que contarles a sus padres sobre su vida sexual. Esta última preocupación también se ha publicado para jóvenes en E.U.A. de Norteamérica ${ }^{23}$.

La diferencia entre especialidades del porcentaje de pacientes con nuevas VG vistos, y la diferencia en el número de visitas de tratamiento entre los sistemas de salud públicos y privados observada podría explicarse porque el sistema público carece de un número suficiente de especialistas, especialmente urólogos, lo que dificulta la programación de los pacientes más de una vez para el tratamiento. Esto demostraría otra inequidad entre los diferentes sistemas, como fue previamente mencionado.

Otro hallazgo interesante en este estudio es que existen diferencias, tanto en las herramientas utilizadas para diagnosticar, como para tratar las VG según sexo de pacientes, especialidades médicas tratantes y el sistema de salud. Las diferencias según sexo y especialidad podrían explicarse por la existencia de diferentes protocolos de manejo entre las diferentes especialidades o a las diferencias en la presentación clínica de las VG en ambos sexos $\mathrm{y}$ en diferentes sitios anatómicos. Sin embargo, en el cuestionario no se diferenció por sitio anatómico (vulva, periné, pene, escroto, ano) o tejido (mucosa, piel) por lo que se requeriría de mayores estudios para conocer la razón exacta de estas diferencias. Las diferencias observadas según sistema de salud utilizado fueron mayores en hombres: en el sistema público de salud se priorizaron los tratamientos de aplicación durante la consulta y la cirugía. La carencia de especialistas en el sistema público, especialmente urólogos, dificultan la programación de los pacientes para más de un control y podría explicar esta priorización; sin embargo, desconocemos si el mayor uso de terapia quirúrgica se pudiese deber, aparte del factor mencionado, a una mayor gravedad de las lesiones en pacientes del sistema público. Respecto al uso de imiquimod, éste tiene un alto costo y no es sufragado por el sistema público. Todas estos podrían ser otros ejemplos de la inequidad en salud en Chile.

\section{Limitaciones del estudio}

La principal limitación del estudio fue la baja tasa de respuesta de los médicos (menos de 10 por ciento de los contactados). Esto podría explicarse en parte por una falta de actualización de los correos electrónicos, por el hecho de que la época del año que usamos para la encuesta era un período habitual de vacaciones en Chile y por un cierto desinterés en participar en estudios de este tipo por parte de los colegas.

Por el contrario, para aquellos que sí respondieron positivamente a la invitación, podría existir un sesgo potencial de "buena voluntad" ("good-will bias") ya que muchos de ellos eran colegas conocidos de los autores. Pese al potencial sesgo de buena voluntad, confiamos en la exactitud de la información provista por parte de los médicos participantes.

Otra limitación fue un error en nuestra adaptación de la bitácora (que solicitó el número diario de pacientes que se vieron) fue que no pedimos el número de pacientes por sexo. Esto dio lugar a una importante limitación, ya que no se pudieron calcular las tasas de prevalencia e incidencia por sexo.

Estas limitaciones pudieran influir en que los resultados no sean representativos de la real prevalencia e incidencia de VG en Chile, ni ser típica para los pacientes atendidos en otras épocas del año.

El estudio de costo-efectividad realizado en Chile previo a la implementación de la vacunación contra el VPH se había enfocado principalmente en el cáncer del cuello del útero, con poco énfasis en los condilomas ${ }^{24}$.

Nuestros hallazgos apoyan la selección de la vacuna tetravalente contra el VPH (VPH 6/11/16/18) para el Programa Nacional de Inmunizaciones (PNI), al considerar una visión más amplia del problema: la infección por VPH como una ITS de alta prevalencia, más que sólo la patología pre-neoplásica y cáncer del cuello del útero asociado a la infección. La prevalencia descrita, la tasa de incidencia de VG en la población atendida y las diferencias en la distribución media de edad y edad por sexo y entre sistemas de salud constituirán una base sobre la cual comparar los datos epidemiológicos en el futuro. El impacto de la introducción de la vacuna tetravalente contra el VPH en el PNI para niñas de 9-10 años en 2014 podría medirse repitiendo el estudio DIACON en una década.

Agradecimientos: Los autores desean agradecer a Ximena Aguilera Sanhueza, del Centro de Epidemiología y Políticas de Salud de la Facultad de Medicina Clínica Alemana - Universidad del Desarrollo por su ayuda en el análisis e interpretación de los resultados. Además agradecer a los médicos que respondieron la encuesta y registraron la bitácora diaria, ya que sin su contribución este estudio no hubiera sido posible: Patricia Apt D., Patricio Barriga P., Eduardo Bustos H., José Miguel Campero P., Andrés Casassas B., Andrea Del Río O., Simón Dujovne C., Luis Ebel S., Alejandra Etchegaray B., Esther Fanjul Á., Carlos Garcés VH, Orietta Gómez H., Mónica Hering S., Ilonka Hoell B., Natalia Jiménez V., Juan Landeros S., Carlos Lobos S., Marcelo Marconi T., Ronnie Martínez B., Eduardo Messina L., Luis Montes M., Nelson 
Muñoz U., Doris Orphanopoulus S., Pablo Oyanedel M., Cristián Palma C., César Paredes M., Diego Peña V., Gonzalo Peña A., Tulio Pino G., Juan Pablo Riquelme P., Tatiana Riveros R., Alexis Rojas P., Myriam Román P., Enrique Roncone D., Fernando San Martín S., Rodrigo Schiaffino O., Conrado Stein G., Roberto Valdés F., María Soledad Vallejo M., Gloria Vergara A., Andrea Vega B., Marcelo Verdugo Ch., Paulina Villaseca D. y Norman Zambrano A.

\section{Resumen}

Introducción: Los condilomas o verrugas genitales (VG) son la infección de transmisión sexual (ITS) más diagnosticada en los centros de ITS en Chile, pero no existen estadísticas poblacionales. Objetivos: Describir la prevalencia de VG en pacientes de 18-60 años que acuden a consulta ambulatoria de dermatología, ginecología y urología; características demográficas de los pacientes y prácticas de diagnóstico y tratamiento. Material y Métodos: A una muestra de especialistas chilenos estratificados por región, población y sexo de pacientes se les proporcionó un diario de registro y aplicó un cuestionario. Resultados: Prevalencia VG grupo total: 2,4\%; en grupo etario 18-34 años: 3,7\%; en grupo etario 35-60 años: $1,29 \%(\mathrm{p}=0,0000)$. La edad media de los pacientes con VG fue 29,4 años en mujeres y 32,7 años en hombres $(p=0,019)$; la distribución por edad fue diferente según sexo y sistema de salud. La inspección visual fue el método diagnóstico más frecuente y la crema de imiquimod el tratamiento más común. Hubo diferencias en el uso de herramientas diagnósticas y terapéuticas según sexo del paciente, especialidad del médico y sistema de salud. Conclusiones: Existe una alta prevalencia de VG, que debería ser tomada en cuenta para planificar las intervenciones de salud pública para abordar este problema.

\section{Referencias bibliográficas}

1.- World Health Organization WHO. Office of Information. Sexually transmitted infections increasing - 250 million new infections annually. WHO Feature. 1990 Dec;(152):1-6. PMID: 12316765.

2.- Patel H, Wagner M, Singhal P, Kothari S. Systematic review of the incidence and prevalence of genital warts. BMC Infect Dis. 2013; 13: 39. doi: 10.1186/1471-2334-13-39.

3.- Castellsagué X, Cohet C, Puig-Tintoré L M, Olmos Acebes L, Salinas J, San Martin M, et al. Epidemiology and cost of treatment of genital warts in Spain. Eur J Public Health 2009; 19 (1): 106-10. doi: 10.1093/eurpub/ckn127.

4.- Syrjänen K, Naud P, Derchain S, Roteli-Martins C, Longatto-Filho A, Tatti S, et al. Comparing PAP smear cytology, aided visual inspection, screening colposcopy, cervicography and HPV testing as optional screening tools in Latin America. Study design and baseline data of the LAMS study. Anticancer Res 2005; 25 (5): 3469-80. PMID: 16101165.

5.- Parkin D M, Almonte M, Bruni L, Cliffordd G, Curadod M P, Piñeros M. Burden and trends of types Specific human papillomavirus infections and related diseases in the Latin America and Caribbean region. Vaccine. 2008; 26 Suppl 11: L1-15. doi: 10.1016/j.vaccine.2008.05.043.

6.- Matos E, Loria D, Amestoy G, Herrera L, Prince M A, Moreno J, et al. Prevalence of human papillomavirus infection among women in Concordia, Argentina: a population-based study. Sex Transm Dis 2003; 30(8): 593-9. doi: 10.1097/01.OLQ.0000085181.25063.6C

7.- Santander E, Fish F, Salvo A, Pacheco G. Normas de manejo y tratamiento de las infecciones de transmisión sexual. Primera parte. Rev Chilena Infectol 2009; 26 (2): 17490 doi: /S0716-10182009000200012.

8.- Gentileza Irene Escribano Dpto VIH MINSAL, 2017.

9.- Giuliano A, Garland S, Salmeron S, Singhal $\mathrm{P}$, Yee K. Designing a multi-country study to estimate burden-of-illness of condyloma acuminate. Presented at: $27^{\text {th }}$ International Papillomavirus Conference \& Clinical Workshops, Berlin. September 17-22, 2011: $\mathrm{P}-02.43$ and personal communication

10.- Kothari S, Yee K, Lesgold S, Ross K M, Feehan M, Garland S, et al. Prevalence of genital warts in gynecology and dermatology practices in Poland. Presented at $28^{\text {th }}$ International Papillomavirus Conference\& Clinical Workshops. November 30-December 6, 2012, San Juan, Puerto Rico. Abstract book Epidemiology/Public Health, page 128.

11. Tsai T F, Hu C H, Yee K S, Kothari S, Lara $\mathrm{N}$, Roset M, et al. Prevalence of genital warts in specialists' practices located in Taiwan. Presented at $28^{\text {th }}$ International Papillomavirus Conference \& Clinical Workshops. November 30-December 6, 2012, San Juan, Puerto Rico. Abstract book Epidemiology/Public Health, page 102

12.- Sagr E R, Aswad S, Sait K H, Hammada K, Yee K S, Kothari S, et al. Prevalence of genital warts in the United Arab Emirates and the Kingdom of Saudi Arabia. Presented at:28 $8^{\text {th }}$ International Papillomavirus Conference \& Clinical Workshops. November 30-December 6, 2012, San Juan, Puerto Rico. [Abstract book Epidemiology/Public Health page 92] 2012, Puerto Rico.

13.- Salmeron P. Comunicación personal y base de datos de MSD.
14.- Departamento de Epidemiología. Ministerio de Salud, Chile. www.minsal.cl

15.- Barros Puente J. Descripción epidemiológica de la mujer adulta sexualmente activa portadora de condilomas de la población adscrita a la red de salud Hospital Padre Hurtado durante un período de observación de 6 meses. Tesis de grado del programa de especialización de Ginecología-Obstetricia de la Universidad del Desarrollo.

16.- Estimaciones y proyecciones de la población de Chile, total país, http://www.censo2017.cl/

17.- Newton-Levinson A, Leichliter J S, ChandraMouli V. Sexually transmitted infection services for adolescents and youth in low- and middleincome countries: perceived and experienced barriers to accessing care. J Adolesc Health 2016; 59 (1): 7-16 https://doi.org/10.1016/j. jadohealth.2016.03.014.

18.- Denison H, Bromhead C, Grainger R, Dennison E, Jutel A. Barriers to sexually transmitted infection testing in New Zealand: a qualitative study Aust N Z J Public Health 2017; 41 (4): 432-7. doi: 10.1111/1753-6405.12680.

19.- Aguilera X, Castillo-Laborde C, Nájera-De Ferrari M, Delgado I, Ibáñez C. Monitoring and evaluating progress towards universal health coverage in Chile. Part of the UHC Measurement Collection. Ploss Medicine 2014; 9 (Sept): 1-3 e1001676 https://doi.org/10.1371/ journal.pmed.1001676.

20.- Castillo-Laborde C, Aguilera-Sanhueza X, Hirmas-Adauy M, Matute I, Delgado-Becerra I, Nájera-De Ferrari M, et al. Health insurance scheme performance and effects on health and health inequalities in Chile. MEDICC Review, 2017; 19 (2-3): 57-64. file:///C:/Users/pepe/ Downloads/mr_591.pdf.

21.- Vlassoff C. Gender differences in determinants 
and consequences of health and illness. J Health Popul Nutr 2007; 25(1): 47-61 PMID: 17615903.

22.- Huneeus A, Deardorff J, Lahiff M, Guendelman S. Type of primary education is associated with condom use at sexual debut among Chilean adolescents. Sex Transm Dis 2014; 41 (5): 306-11. doi: 10.1097/ OLQ.0000000000000122.
23.- Leichliter J S, Copen C, Dittus P J. Confidentiality issues and use of sexually transmitted disease services among sexually experienced persons aged 15-25 years-United States, 2013-2015 MMWR Morb Mortal Wkly Rep 2017; 66 (9): 237-41.

24.- Castillo M, Castillo C, Aravena M, Departamento de Economía de la Salud, División de Planificación Sanitaria,
Subsecretaría de Salud Pública, MINSAL, 2011. Sistematización de la Información sobre cáncer cérvico uterino en Chile: revisión y análisis de costo-efectividad de la vacuna contra VPH. Disponible en http://ciperchile.cl/ wp-content/uploads/Papiloma_Sistematizacion de_la_Informacion_sobre_ConcerCrvico_ Uterino_Chile2011Minsal.pdf (Último acceso el 22 de enero de 2018). 